\title{
Cerebral Amyloid Positivity Prediction Models Using Clinical Data in Subjects With Mild Cognitive Impairment and Dementia
}

\author{
Soo Hyun Joo and Chang Uk Lee ${ }^{凶}$ \\ Department of Psychiatry, Seoul St. Mary's Hospital, College of Medicine, The Catholic University of Korea, Seoul, Republic of Korea
}

\begin{abstract}
Objective Due to high cost of amyloid imaging, its use of amyloid imaging to confirm amyloid pathology is limited in clinical practice. It is of importance to develop a model to predict cerebral amyloid positivity using clinical data obtained from a memory clinic.

Methods A total of 410 participants who had symptom of subjective cognitive decline and underwent amyloid PET and apolipoprotein $\varepsilon$ (APOE) genotyping were retrospectively enrolled from January 2016 to January 2019. Models for cerebral amyloid positivity prediction were developed in all subjects, mild cognitive impairment (MCI) subjects, and Alzheimer's disease (AD) dementia subjects through multivariate logistic regression analysis. The performance of the models was assessed using receiver operating characteristic (ROC) curve analysis and the area under the curve (AUC) values.

Results Age, sex, years of education, body mass index (BMI), APOE4, and mini mental state examination score (MMSE) were selected for the final model for all subjects. The AUC value of the ROC curve was 0.775. Age, sex, years of education, BMI, and APOE4 were selected for the final model for MCI subjects. The AUC value was 0.735. Age, sex, years of education, BMI, APOE4, MMSE, and history of hypertension were selected for the final model for AD dementia subjects. The AUC value was 0.845 .

Conclusion This study found that models using clinical data can predict cerebral amyloid positivity according to cognitive status. These models can be useful as a screening tool predict cerebral amyloid deposition in cognitively impaired patients in a memory clinic.
\end{abstract}

Psychiatry Investig 2021;18(9):864-870

Keywords Amyloid; Apolipoprotein E; Cognition; Alzheimer's disease; Dementia.

\section{INTRODUCTION}

The global population of Alzheimer's disease $(\mathrm{AD})$ patients is about 35 million people and expected to be 115.3 million people in 2050. ${ }^{1}$ Pathognomonic findings in pathologies of AD are characterized by amyloid plaque and neurofibrillary tangles, consisting of abnormal accumulation of beta-amyloid $(A \beta)$ protein and tau proteins, respectively, in the brain. These two abnormal proteins accumulate about 15 years before onset of symptoms including complaints of cognitive decline. Given that there are no disease modifying treatments in $\mathrm{AD}$, it is important to assess the risk of $\mathrm{AD}$ and detect the disease at the early stage. ${ }^{2}$

Received: March 22, 2021 Revised: July 1, 2021

Accepted: July 7, 2021

$\triangle$ Correspondence: Chang Uk Lee, $\mathrm{MD}, \mathrm{PhD}$

Department of Psychiatry, Seoul St. Mary's Hospital, College of Medicine, The Catholic University of Korea, 222 Banpo-daero, Seocho-gu, Seoul 06591, Republic of Korea

Tel: +82-2-2258-6082, Fax: +82-2-594-3870, E-mail: jihan@catholic.ac.kr

(a) This is an Open Access article distributed under the terms of the Creative Commons Attribution Non-Commercial License (https://creativecommons.org/licenses/bync/4.0) which permits unrestricted non-commercial use, distribution, and reproduction in any medium, provided the original work is properly cited.
$\mathrm{AD}$ has been diagnosed based on clinical diagnostic criteria in most clinical practice. However, the diagnostic method in $\mathrm{AD}$ based on clinical diagnostic criteria has limitations, showing low sensitivity (71\% to $81 \%$ ) and specificity (about $70 \%)$, since confirmation of $\mathrm{AD}$ is based on the histopathologic findings. ${ }^{3} \mathrm{Ce}-$ rebrospinal fluid (CSF) A $\beta$ examination is a useful, low-cost fluid biomarker for cerebral amyloid $A \beta$ pathology. However, is use in clinics is limited because the test is not only invasive, but also inapplicable in patients with increased intracranial pressure, coagulopathy, or current use of anticoagulant. As amyloid positron emission tomography (PET), non-invasive imaging marker of $A \beta$ in the brain, has been developed, it is possible to confirm $\mathrm{AD}$ without post-mortem autopsy. ${ }^{4}$ However, its accessibility is low in the real clinical settings due to high cost.

In 2013, the Alzheimer's Association and the Society of Nuclear Medicine and Molecular Imaging convened the Amyloid Imaging Taskforce to gather empirical evidence to support the clinical utility of amyloid imaging. Patients who have persistent unexplained mild cognitive impairment (MCI), patients with atypical presentations of $\mathrm{AD}$, and patients who develop pro- 
gressive neurocognitive disorder (NCD) symptoms at an atypically early age are more likely to benefit from amyloid imaging. ${ }^{5}$ However, when we apply the guideline in a memory clinic, more specific guidelines, including demographics or genetics, for conducting amyloid PET are needed. Although several previous studies have made prediction models for cerebral amyloid positivity, there were limitations in applying clinically unavailable variables to the model or creating a model for one disorder such as $\mathrm{MCI}$ or $\mathrm{AD}$. We aimed to develop amyloid prediction models in subjects with varying cognitive status using various clinical data routinely obtained in a regular clinical practice. We will develop three models: one for all subjects suffering from cognitive decline, one for MCI subjects, and one for $\mathrm{AD}$ dementia subjects. In addition, we aimed to assess the performance pf amyloid prediction models.

\section{METHODS}

\section{Study population}

A total of 410 patients with various diagnosis, 410 participants with MCI or $\mathrm{AD}$ were enrolled in this study from a cohort of cognitive impairment in the Seoul St. Mary's Hospital, Catholic Medical Center. This study included participants who underwent both amyloid PET and apolipoprotein $\varepsilon$ (APOE) genotyping from January 2016 to January 2019.

\section{Diagnostic assessments}

Detailed history taking, comprehensive physical examinations, including body weight, height, and blood pressure (BP), laboratory tests, and brain imaging, such as magnetic resonance imaging (MRI), or computed tomography (CT), were performed in all of the subjects. With regard to clinical history, age, sex, years of education, family history of dementia, and past medical history, including diabetes mellitus (DM), hypertension (HTN) were collected. Body mass index (BMI) was calculated by the body weight divided by the square of the body height. To exclude other causes of cognitive impairment, a thyroid function test (thyroid stimulating hormone), lipid profile (total cholesterol), and vitamin B12 were assessed using blood. APOE genotype was determined by PCR using blood samples in EDTA tubes. Subjects with at least one $\varepsilon 4$ allele were classified as APOE4 positive. Mini mental state examination (MMSE) and sum of the box score for clinical dementia rating (CDR-SB) were used to assess global cognition in all participants. In addition, a neuropsychological battery such as the Seoul Neuropsychological Screening Battery $(\mathrm{SNSB})^{6}$ or the Korean version of the Consortium to Establish a Registry for Alzheimer's Disease (CERAD$\mathrm{K})^{7}$ was used for assessment of multiple cognitive function.

Clinical diagnosis was made based on the clinical diagnostic criteria for subjective memory complaint (SMC), MCI, Al- zheimer's dementia, frontotemporal dementia (FTD), vascular dementia (VaD), and dementia with Lewy body (DLB). ${ }^{8-14}$ We included $\mathrm{MCI}$ and $\mathrm{AD}$ patients in this study.

\section{Amyloid PET/CT scan}

Amyloid PET/CT scans were performed on a Discovery PET/ CT 710 (GE Medical Systems, Milwaukee, WI, USA) or a Biograph TruePoint 40 PET/CT (Siemens Medical Solutions USA, Knoxville, TN, USA). Here, 18F-florbetaben and 18F-flutemetamol were used as $18 \mathrm{~F}$-labeled $\mathrm{A} \beta$ targeting tracers. Patients received an intravenous injection of $8.0 \mathrm{mCi}$ of $18 \mathrm{~F}$-florbetaben or $5.0 \mathrm{mCi}$ of $18 \mathrm{~F}$-flutemetamol and rested in a waiting room. Image acquisition started approximately 90 minutes after the injection, with a scan duration of 20 minutes.

The 18F-florbetaben PET and flutemetamol PET scans were visually rated as either amyloid-positive or amyloid-negative by the trained nuclear medicine physician. Interpretation of the 18F-florbetaben PET images was achieved by visually comparing the activity in the cortical gray matter with the activity in the adjacent cortical white matter. Brain regions of the-lateral temporal cortex, frontal cortex, posterior cingulate cortex/precuneus, and parietal cortex-should be systematically and cortical tracer uptake at any of the cortical target regions, while a negative scan indicates good grey-white matter contrast, with no tracer uptake at target regions. ${ }^{15}$ The regions of interest of 18F-flutemetamol PET scans were the frontal lobe, posterior cingulate cortex/precuneus, lateral temporal lobe, parietal lobe, and striatum. Negative scans show more radioactivity in white matter than in grey matter, creating clear grey-white matter contrast. Positive scans show at least one cortical region with reduction or loss of the normally distinct grey-white matter contrast. ${ }^{16}$

\section{Development of cerebral amyloid prediction models}

The following variables were used for developing amyloid prediction models; age, sex, years of education, BMI, family history of dementia, history of hypertension or DM, MMSE, CDR-SB, APOE4 genotype, total cholesterol, TSH, vitamin B12, blood pressure and clinical diagnosis. These variables were included because they are well-known risk factors for dementia.

\section{Statistical analysis}

Continuous variables, including age, education (years), BMI, the MMSE, the CDR-SB, systolic and diastolic BP, TSH, total cholesterol, and vitamin B12, are presented with the mean and standard error (SE). Independent t-test was used to compare subjects with amyloid deposition and those without amyloid deposition. Categorical variables, including sex, family history of dementia, DM, HTN, APOE genotype, and clinical diagnosis, are presented with number and percentage. Chi-square test was used to compare between group with amyloid deposition 
and group without amyloid deposition. $\mathrm{p}$ value $<0.05$ was considered as statistically significant.

Through multivariate logistic regression analysis using a forward conditional method, we developed final models for cerebral amyloid positivity prediction. Age, sex, and year of education were entered as fixed variables. Sex, the categorical variable, was coded as 1 for male and 2 for female. Other variables were sequentially entered into the model using the forward likelihood ratio (LR) method. Odds ratios (ORs) and 95\% confidence interval (CIs) were used to identify associations between clinical variables and amyloid positivity. We developed three cerebral amyloid prediction models according to cognitive status for all subjects, MCI subjects, and AD dementia subjects. The discriminative power of the cerebral amyloid prediction models was assessed using receiver operating characteristic (ROC) curve analysis and the area under the curve (AUC) values. Also, we produced simple models for cerebral amyloid prediction using logistic regression that included age, sex, years of education, and one of the other variables in the final model to compare the final models with each of the simple models. Additionally, we explored how the Youden index, sensitivity, specificity, positive predictive value (PPV), and negative predictive value (NPV) increases from 0.1 to 0.9 by 0.1 unit and presented the optimal cutoff point for probability for being amyloid positive (Supplementary Tables 1-3 in the online-only Data Supplement). All statistical analyses were performed using SAS version 9.2 (SAS Institute Inc., Cary, NC, USA).

\section{Ethics approval}

The Institutional Review Board of the Catholic Medical Center approved this study protocol (KC20RESASI0639).

\section{RESULTS}

\section{Demographic characteristics}

The characteristics of the 410 subjects according to amyloid positivity are summarized in Table 1 . The proportion of amyloid PET-negative patients was $45.9 \%(n=188)$, while there were $54.1 \%(\mathrm{n}=222)$ amyloid PET-positive patients. The patient diagnoses were $297 \mathrm{MCI}, 113 \mathrm{AD}$ dementia. The amyloid PET-positive patients were older than amyloid PET-negative patients. The amyloid PET-positive group contained $70.3 \%$ females, while the amyloid PET-negative group was $61.2 \%$ females. BMI was lower in the amyloid PET-positive group than in the amyloid PETnegative group. In the amyloid PET-positive group, 53.6\% of patients were APOE4-positive, while $17.0 \%$ of the patients in

Table 1. Clinical characteristics of all subjects according to results of amyloid PET

\begin{tabular}{|c|c|c|c|}
\hline & $\mathrm{Am}$ & & n-yalue \\
\hline & Negative & Positive & $P$-vaicue \\
\hline Number & 188 & 222 & \\
\hline Age, years & $74.48 \pm 8.54$ & $76.01 \pm 8.64$ & 0.073 \\
\hline Female, N (\%) & $115(61.17)$ & $156(70.27)$ & 0.052 \\
\hline BMI, $\mathrm{kg} / \mathrm{m}^{2}$ & $23.73 \pm 3.31$ & $23.16 \pm 3.14$ & 0.079 \\
\hline Education, years & $11.70 \pm 4.81$ & $10.91 \pm 5.10$ & 0.105 \\
\hline APOE4-positive, N (\%) & $32(17.02)$ & $119(53.60)$ & $<0.001$ \\
\hline Family history of dementia, N (\%) & $46(25.41)$ & $64(30.77)$ & 0.242 \\
\hline Hypertension, $\mathrm{N}(\%)$ & $92(48.94)$ & $102(45.95)$ & 0.546 \\
\hline Diabetes mellitus, N (\%) & $59(31.38)$ & $53(23.87)$ & 0.089 \\
\hline Diagnosis, $\mathrm{N}(\%)$ & & & $<0.001$ \\
\hline MCI & $160(85.11)$ & $137(61.71)$ & \\
\hline $\mathrm{AD}$ dementia & $28(14.89)$ & $85(38.29)$ & \\
\hline MMSE score & $24.69 \pm 3.63$ & $21.87 \pm 5.54$ & $<0.001$ \\
\hline CDR-SB score & $2.5 \pm 2.32$ & $4.09 \pm 3.64$ & $<0.001$ \\
\hline Systolic blood pressure, $\mathrm{mm} \mathrm{Hg}$ & $126.35 \pm 17.34$ & $128.77 \pm 16.75$ & 0.191 \\
\hline Diastolic blood pressure, $\mathrm{mm} \mathrm{Hg}$ & $72.54 \pm 10.56$ & $73.40 \pm 11.25$ & 0.469 \\
\hline $\mathrm{TSH}, \mathrm{mIU} / \mathrm{L}$ & $2.75 \pm 7.60$ & $2.64 \pm 2.89$ & 0.859 \\
\hline Total cholesterol, mg/dL & $178.88 \pm 41.05$ & $186.04 \pm 40.54$ & 0.111 \\
\hline Vitamin B12, pg/mL & $823.89 \pm 768.07$ & $751.12 \pm 552.97$ & 0.344 \\
\hline
\end{tabular}

PET, positron emission tomography; BMI, body mass index; APOE, apolipoprotein $\varepsilon$; MCI, mild cognitive impairment; AD, Alzheimer's disease, MMSE, mini-mental state examination; CDR-SB, clinical dementia rating sum of box; TSH, thyroid stimulating hormone 
the amyloid PET-negative group were APOE4-positive. The patients in the amyloid PET-positive group had lower cognitive function than the patients in the amyloid PET negative group, which was shown by lower MMSE and higher CDR-SB score in the amyloid PET-positive group. In blood chemistry, total cholesterol was higher in the amyloid PET-positive group than in the amyloid PET-negative group.

\section{Cerebral amyloid prediction models in all subjects, MCI subjects, and AD dementia subjects}

We developed three final models using multivariate logistic regression analysis in all subjects, $\mathrm{MCI}$ subjects, and $\mathrm{AD}$ dementia subjects.

$\mathrm{P}=$ probability for being cerebral amyloid positive

$\mathrm{P}_{\text {case }}=\left(\exp \left[\right.\right.$ Logit $\left.\left._{\text {case }}\right]\right) /\left(1+\exp \left[\right.\right.$ Logit $\left.\left._{\text {case }}\right]\right)$

$\operatorname{Logit}_{\text {all }}=\beta 0+\beta 1$ (age) $+\beta 2$ (sex) $+\beta 3$ (years of education) $+\beta 4$ (APOE4 positivity) $+\beta 5$ (MMSE score) $(\beta 0=0.158, \beta 1=0.029$, $\beta 2=0.247, \beta 3=0.034, \beta 4=1.937, \beta 5=-0.155)$

$\operatorname{Logit}_{\mathrm{MCI}}=\beta 0+\beta 1$ (age) $+\beta 2$ (sex) $+\beta 3$ (years of education) $+\beta 4$ (APOE4 positivity) $(\beta 0=-3.668, \beta 1=0.037, \beta 2=-0.016, \beta 3=0.004$, $\beta 4=1.915)$

$\operatorname{Logit}_{\mathrm{AD}}=\beta 0+\beta 1$ (age) $+\beta 2($ sex $)+\beta 3$ (years of education $)+\beta 4$ (APOE4 positivity) $+\beta 5$ (MMSE score) $+\beta 6$ (history of hypertension) $(\beta 0=-0.012, \beta 1=0.039, \beta 2=1.043, \beta 3=0.075, \beta 4=2.631$, $\beta 5=-0.198, \beta 6=-1.615)$

The first model was to predict cerebral amyloid positivity for all subjects with subjective cognitive decline in our memory clinic. Age, sex, years of education, BMI, APOE4, and MMSE score were selected for the final model for all subjects (Table 2). The AUC value of the ROC curve for the developed model was 0.775 (95\% confidence interval $[\mathrm{CI}]=0.731$ to 0.820 ) (Figure 1 ). The second model was to predict cerebral amyloid positivity for MCI subjects. Age, sex, years of education, BMI, and APOE4 were selected for the final model for MCI subjects (Table 3). The AUC value of the ROC curve for the developed model was 0.735 (95\% CI=0.676 to 0.793) (Figure 1). The third model was to predict cerebral amyloid positivity for $\mathrm{AD}$ subjects. Age, sex, years of education, BMI, APOE4, MMSE score, and history of

Table 2. The final and simpler models predicting cerebral amyloid positivity in all subjects

\begin{tabular}{|c|c|c|c|c|}
\hline Variable & Beta & Wald & OR (95\% CI) & p-value \\
\hline \multicolumn{5}{|l|}{ Final model } \\
\hline Age & 0.029 & 4.374 & $1.030(1.002,1.058)$ & 0.037 \\
\hline Sex & 0.247 & 0.953 & $1.280(0.780,2.100)$ & 0.329 \\
\hline Education & 0.034 & 1.653 & $1.034(0.983,1.088)$ & 0.199 \\
\hline APOE4 & 1.937 & 55.879 & $6.939(4.176,11.532)$ & $<0.0001$ \\
\hline MMSE & -0.155 & 27.068 & $0.857(0.808,0.908)$ & $<0.0001$ \\
\hline Intercept & 0.158 & & & \\
\hline R-square* & 0.302 & & & \\
\hline \multicolumn{5}{|c|}{ APOE4-only model } \\
\hline Age & 0.038 & 8.213 & $1.038(1.012,1.066)$ & 0.004 \\
\hline Sex & 0.203 & 0.694 & $1.225(0.760,1.974)$ & 0.405 \\
\hline Education & -0.010 & 0.176 & $0.990(0.946,1.037)$ & 0.675 \\
\hline APOE4 & 1.826 & 54.903 & $6.208(3.830,10.062)$ & $<0.0001$ \\
\hline Intercept & -3.517 & & & \\
\hline R-square* & 0.215 & & & \\
\hline \multicolumn{5}{|c|}{ MMSE-only model } \\
\hline Age & 0.009 & 0.564 & $1.009(0.985,1.034)$ & 0.453 \\
\hline Sex & 0.354 & 2.344 & $1.425(0.905,2.243)$ & 0.126 \\
\hline Education & 0.021 & 0.776 & $1.021(0.975,1.070)$ & 0.378 \\
\hline MMSE & -0.138 & 25.617 & $0.871(0.826,0.919)$ & $<0.0001$ \\
\hline Intercept & 1.883 & 2.089 & & 0.148 \\
\hline R-square* & 0.122 & & & \\
\hline
\end{tabular}

*Nagelkerke R-square. OR, odds ratio; CI, confidence interval; APOE, apolipoprotein $\varepsilon$; MMSE, mini-mental state examination
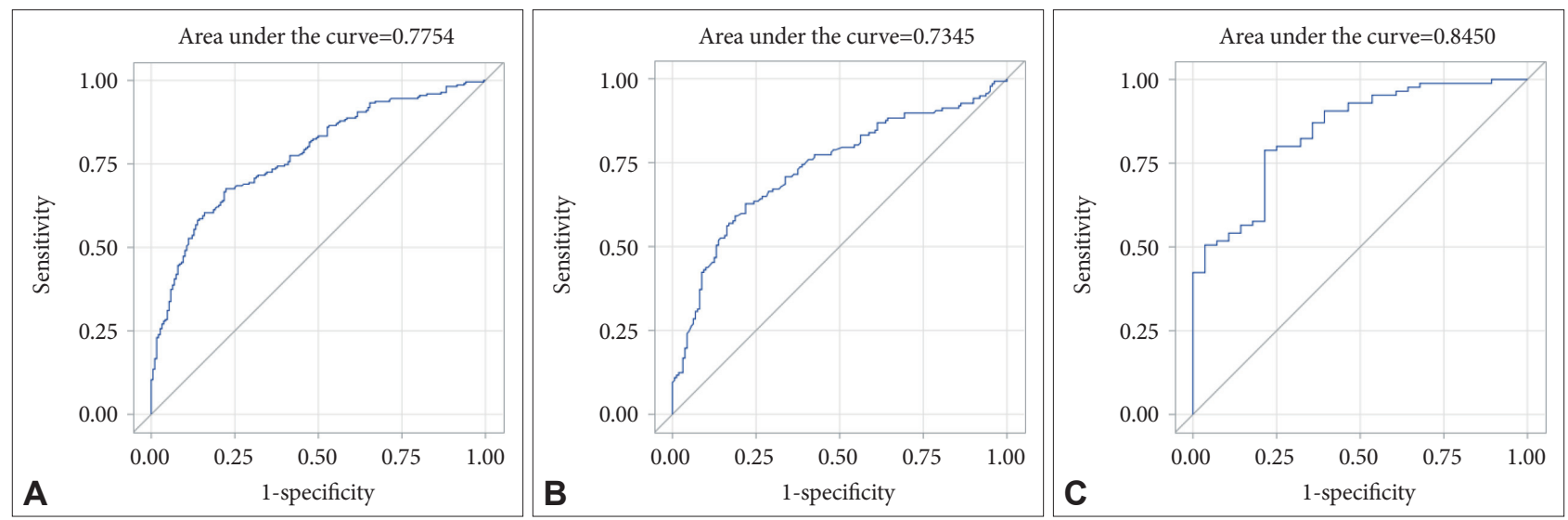

Figure 1. Receiver operating characteristic curves for the final and the three prediction models for cerebral amyloid positivity. (A) All subjects, (B) mild cognitive impairment subjects, and (C) Alzheimer's disease dementia subjects. 
Table 3. The final model predicting cerebral amyloid positivity in $\mathrm{MCl}$ subjects

\begin{tabular}{lrrcc}
\hline Final model & Beta & Wald & OR $(95 \%$ CI $)$ & p-value \\
\hline Age & 0.037 & 5.679 & $1.038(1.007,1.071)$ & 0.017 \\
Sex & -0.016 & 0.003 & $0.985(0.565,1.714)$ & 0.956 \\
Education & 0.004 & 0.018 & $1.004(0.949,1.062)$ & 0.893 \\
APOE4 & 1.915 & 46.405 & $6.784(3.911,11.768)$ & $<0.0001$ \\
Intercept & -3.668 & & & \\
R-square* & 0.227 & & & \\
\hline
\end{tabular}

*Nagelkerke R-square. MCI, mild cognitive impairment; OR, odds ratio; $\mathrm{CI}$, confidence interval; $\mathrm{APOE}$, apolipoprotein $\varepsilon$

Table 4. The final and simpler models predicting cerebral amyloid positivity in $A D$ dementia subjects

\begin{tabular}{|c|c|c|c|c|c|c|c|c|}
\hline Variable & Beta & Wald & OR (95\% CI) & $\overline{p \text {-value }}$ & Hypertension-only model & 0.724 & 0.615 & 0.832 \\
\hline
\end{tabular}

Final model

$\begin{array}{lllll}\text { Age } & 0.039 & 1.016 & 1.040(0.964,1.122) & 0.314\end{array}$

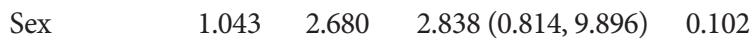

$\begin{array}{lllll}\text { Education } \quad 0.075 & 1.724 & 1.077(0.964,1.204) & 0.189\end{array}$

$\begin{array}{lllll}\text { APOE4 } & 2.631 & 9.339 & 13.890(2.569,75.089) & 0.002\end{array}$

$\begin{array}{lllll}\text { MMSE } & -0.198 & 8.795 & 0.820(0.720,0.935) & 0.003\end{array}$

Hypertension $-1.615 \quad 6.443 \quad 0.199(0.057,0.692) \quad 0.011$

Intercept $\quad-0.012$

R-square* $\quad 0.429$

APOE4-only model

$\begin{array}{lrrcr}\text { Age } & -0.002 & 0.003 & 0.998(0.939,1.061) & 0.958 \\ \text { Sex } & 1.213 & 4.271 & 3.365(1.065,10.634) & 0.039 \\ \text { Education } & 0.050 & 0.943 & 1.051(0.950,1.164) & 0.332 \\ \text { APOE4 } & 2.453 & 9.888 & 11.619(2.519,53.589) & 0.002 \\ \text { Intercept } & -1.831 & & & \\ \text { R-square* } & 0.259 & & & \end{array}$

MMSE-only model

$\begin{array}{lrrrr}\text { Age } & -0.008 & 0.065 & 0.992(0.932,1.056) & 0.799 \\ \text { Sex } & 1.061 & 3.851 & 2.890(1.001,8.342) & 0.050 \\ \text { Education } & 0.048 & 0.865 & 1.049(0.948,1.162) & 0.352 \\ \text { MMSE } & -0.125 & 6.066 & 0.882(0.798,0.975) & 0.014 \\ \text { Intercept } & 1.955 & & & \\ \text { R-square* } & 0.160 & & & \end{array}$

Hypertension-only model

\begin{tabular}{lrrrr} 
Age & 0.006 & 0.036 & $1.006(0.942,1.075)$ & 0.849 \\
Sex & 0.987 & 3.105 & $2.684(0.895,8.049)$ & 0.078 \\
Education & 0.017 & 0.115 & $1.017(0.924,1.119)$ & 0.735 \\
Hypertension & -1.579 & 7.940 & $0.206(0.069,0.618)$ & 0.005 \\
Intercept & -0.143 & & & \\
R-square* & 0.185 & & & \\
\hline
\end{tabular}

*Nagelkerke R-square. AD, Alzheimer's disease; OR, odds ratio; $\mathrm{CI}$, confidence interval; APOE, apolipoprotein $\varepsilon$; MMSE, minimental state examination
Table 5. The AUC values of ROC curves for the three prediction models for cerebral amyloid positivity

\begin{tabular}{lccc}
\hline \multicolumn{1}{c}{ Model } & AUC & Lower & Upper \\
\hline All subjects & & & \\
$\quad$ Final model & 0.775 & 0.731 & 0.820 \\
$\quad$ APOE4-only model & 0.730 & 0.681 & 0.779 \\
$\quad$ MMSE-only model & 0.663 & 0.611 & 0.715 \\
MCI subjects & & & \\
$\quad$ Final model & 0.735 & 0.676 & 0.793 \\
AD subjects & & & \\
$\quad$ Final model & 0.845 & 0.765 & 0.925 \\
$\quad$ APOE4-only model & 0.747 & 0.652 & 0.842 \\
$\quad \begin{array}{l}\text { MMSE-only model } \\
\text { Hypertension-only model }\end{array}$ & 0.710 & 0.603 & 0.816 \\
\hline AUC area & 0.615 & 0.832 \\
\hline
\end{tabular}
tic; APOE, apolipoprotein $\varepsilon$; MMSE, mini-mental state examination; $\mathrm{MCI}$, mild cognitive impairment; $\mathrm{AD}$, Alzheimer's disease

hypertension were selected for the final model for $\mathrm{AD}$ subjects (Table 4). The AUC value of the ROC curve for the developed model was 0.845 (95\% CI=0.765 to 0.925 ) (Figure 1). We created simple models for cerebral amyloid prediction that included age, sex, years of education, and BMI as fixed variables and one of the other variables in the final model (APOE4, and MMSE in the all subjects model, and APOE4, MMSE, and history of hypertension in the $\mathrm{AD}$ dementia model) to compare the final models and each of the simple models (Tables 2 and 4). The AUC value for the all subjects model was 0.730 for the APOE4-only model, and 0.663 for the MMSE-only model. The AUC value for the AD dementia model was 0.747 for the APOE4-only model, 0.710 for the MMSE-only model, and 0.724 for the hypertension-only model. Statistical comparisons of the AUC values between the final models and each of the simple models show that the final model had a significantly larger AUC value than any simple model (Table 5).

\section{DISCUSSION}

The major findings of the present study were as follows. First, we found that older age, female sex, lower BMI, APOE4 genotype, lower MMSE score, higher CDR-SB score, and elevated blood total cholesterol independently predicted cerebral amyloid positivity in all subjects. Second, we developed models to predict the risk of cerebral amyloid positivity in all subjects, MCI subjects, and $\mathrm{AD}$ dementia subjects. Additionally we found superiority of ability to predict amyloid positivity by comparing final models and simple models.

Several previous studies have made prediction models for cerebral amyloid positivity. Bahar-Fuchs et al. ${ }^{17}$ focused on neuropsychological tests, and they reported that episodic memo- 
ry impairment was related to cerebral amyloid positivity in 45 MCI patients. Also, several prediction models focused on bloodbased biomarkers have been reported. ${ }^{18-21}$ Among these studies, three used plasma $A \beta$ for predicting cerebral amyloid deposits. ${ }^{19-21}$ Unfortunately, the blood-based biomarkers presented in these studies are limited because they are not commonly used in clinical practice. Haghighi et al. ${ }^{22}$ developed a cerebral amyloid prediction model by integrating neuropsychological tests and plasma-based biomarkers in $168 \mathrm{MCI}$ and 50 cognitively normal subjects. However, the study also included blood-based biomarkers that are not routinely available in clinical practice. Lee et al. ${ }^{23}$ proposed a cerebral amyloid prediction model using commonly available clinical information on age, sex, years of education, APOE4, history of hypertension, and word list recall test score in $107 \mathrm{MCI}$ and 69 early AD dementia patients for the KBASE cohort, and in $332 \mathrm{MCI}$ and $144 \mathrm{AD}$ dementia patients for the ADNI-2 cohort. In contrast, we developed three models: one for all subjects suffering from cognitive decline, one for MCI subjects, and one for $\mathrm{AD}$ dementia subjects. Also, our study included blood tests, and neuropsychological measurements of the MMSE and CDR-SB, which are commonly used during dementia evaluations in clinical practice. Therefore, our models make it possible to easily predict cerebral amyloid positivity using data obtained by routinely in clinical settings. Also, each model could be applied according to cognitive level.

In the models we developed, the AUC values were 0.775 for all subjects, 0.735 for MCI subjects, and 0.845 for $\mathrm{AD}$ dementia subjects. This means that the models can predict the positive rates of cerebral amyloid PET in 77.5\%, 73.5\%, and 84.5\%, respectively. The all subjects model was applied to patients whose objective cognitive function level was not identified. The possibility of amyloid PET positivity was $77.5 \%$ in the subjects who were elderly, female, and had a low MMSE score, and had the APOE4 gene. The MCI model was applied to patients diagnosed with MCI. The possibility of amyloid PET positivity was $73.5 \%$ in subjects who were elderly, and had the APOE4 gene. These patients may need to undergo an amyloid PET or prepare for an early preventive intervention. In addition, the AD model was applied to patients probable AD dementia by NIAAA diagnostic guideline. The possibility of amyloid PET positivity was $84.5 \%$ in subjects who were elderly, had low MMSE score, the APOE4 gene, and no history of hypertension. This result can help us develop additional diagnostic or therapeutic plans.

In the present study, APOE4 status was repeatedly associated with cerebral amyloid positivity in the three models for all subjects, MCI subjects, and AD dementia subjects. Inheritance of the $\varepsilon 4$ allele of the APOE gene is the strongest genetic risk factor for $\mathrm{AD}$. The interaction between APOE and $\mathrm{A} \beta$ plays a key role in $\mathrm{AD}$ pathogenesis. Previous studies revealed that the APOE- $\mathrm{A} \beta$ interaction regulates $\mathrm{A} \beta$ aggregation and clearance and therefore directly influences development of amyloid plaques, congophilic amyloid angiopathy, and the subsequent tau-related pathology. ${ }^{24}$ We found that the APOE4 gene had the strongest association with cerebral amyloid positivity among all variables. Therefore, since it is possible for patients with the APOE4 gene to accumulate cerebral $A \beta$, it is necessary to implement amyloid PET early or to establish a therapeutic intervention for all levels of cognitive function. MMSE scores were included in the final models for all subjects and for $\mathrm{AD}$ dementia subjects. The MMSE test is one of the simplest and most widely used screening tests for patients complaining of cognitive decline. We found that a lower MMSE score is a predictor for cerebral amyloid positivity in all subjects and in $\mathrm{AD}$ dementia subjects. History of hypertension was negatively correlated with cerebral amyloid positivity in $\mathrm{AD}$ dementia subjects. Demented individuals with a hypertension were less likely to be cerebral amyloid deposits than those without a hypertension. Since chronic hypertension can reduce brain reserve through overall or regional brain atrophy, ${ }^{25-28}$ individuals with a hypertension are more likely to be cognitively impaired with a relatively lower amyloid accumulation. Although APOE4, MMSE score, and history of hypertension were each significantly related to cerebral amyloid positivity, the final model with all three variables showed a superior screening ability compared to the simper models with only one variable in $\mathrm{AD}$ dementia subjects.

This study has several limitations. First, although our study included patients with vascular dementia, Lewy body dementia, frontotemporal dementia, and other types of dementia, we could not develop models to predict cerebral amyloid positivity for each type of dementia due to the small number of subjects. Second, it is possible that clinicians have applied amyloid PET to patients who are likely to be cerebral amyloid-positive. Third, since the same neuropsychological battery such as SNSB or CERAD-K was not applied uniformly, we could not include it in the analysis except for MMSE and CDR-SB results. Fourth, two types of $18 \mathrm{~F}$-labeled amyloid- $\beta$ targeting tracers were applied to the study subjects, but these were not considered when developing the models. Nevertheless, this is the first study to developed three cerebral amyloid prediction models according to the level of cognitive function by integrating clinically obtained demographic, hematologic, and neuropsychiatric tests data.

In conclusion, we developed cerebral amyloid prediction models with variables that are routinely collected in clinical practice for subjects complaining of cognitive decline. This study demonstrated that integration of demographic variables, neuropsychological measurements, and blood-based markers significantly improved prediction accuracy. Furthermore, our developed models have the potential to be widely used as screening tools, because we created cerebral amyloid prediction models 
with data obtained routinely in clinical settings. We hope that early prediction of cerebral amyloid positivity through data commonly used in a memory clinic, will be helpful for screening cognitively impaired patients with high probability of cerebral amyloid deposition.

\section{Supplementary Materials}

The online-only Data Supplement is available with this article at https://doi.org/10.30773/pi.2021.0104.

\section{Availability of Data and Material}

The datasets generated or analyzed during the study are available from the corresponding author on reasonable request.

\section{Conflicts of Interest}

The authors have no potential conflicts of interest to disclose.

\section{Author Contributions}

Conceptualization: Chang Uk Lee. Data curation: Soo Hyun Joo. Formal analysis: Chang Uk Lee. Investigation: Soo Hyun Joo. Methodology: Soo Hyun Joo. Resources: Soo Hyun Joo. Software: Soo Hyun Joo. Supervision: Chang Uk Lee. Validation: Chang Uk Lee. Visualization: Soo Hyun Joo. Writing-original draft: Soo Hyun Joo. Writing_review \& editing: Chang Uk Lee, Soo Hyun Joo.

\section{ORCID iDs}

Soo Hyun Joo https://orcid.org/0000-0003-1390-3623

Chang Uk Lee https://orcid.org/0000-0001-6398-7330

\section{Funding Statement}

None.

\section{REFERENCES}

1. Alzheimer's Association. 2013 Alzheimer's disease facts and figures. Alzheimers Dement 2013;9:208-245.

2. Bateman RJ, Xiong C, Benzinger TL, Fagan AM, Goate A, Fox NC, et al. Clinical and biomarker changes in dominantly inherited Alzheimer's disease. N Engl J Med 2012;367:795-804.

3. Beach TG, Monsell SE, Phillips LE, Kukull W. Accuracy of the clinical diagnosis of Alzheimer disease at National Institute on Aging Alzheimer Disease Centers, 2005-2010. J Neuropathol Exp Neurol 2012;71:266-273.

4. Ossenkoppele R, Prins ND, Pijnenburg YA, Lemstra AW, van der Flier WM, Adriaanse SF, et al. Impact of molecular imaging on the diagnostic process in a memory clinic. Alzheimers Dement 2013;9:414-421.

5. Johnson KA, Minoshima S, Bohnen NI, Donohoe KJ, Foster NL, Herscovitch P, et al. Appropriate use criteria for amyloid PET: a report of the Amyloid Imaging Task Force, the Society of Nuclear Medicine and Molecular Imaging, and the Alzheimer's Association. Alzheimers Dement 2013;9:e-1-16.

6. Ahn HJ, Chin J, Park A, Lee BH, Suh MK, Seo SW, et al. Seoul Neuropsychological Screening Battery-dementia version (SNSB-D): a useful tool for assessing and monitoring cognitive impairments in dementia patients. J Korean Med Sci 2010;25:1071-1076.

7. Lee JH, Lee KU, Lee DY, Kim KW, Jhoo JH, Kim JH, et al. Development of the Korean version of the Consortium to Establish a Registry for Alzheimer's Disease Assessment Packet (CERAD-K): clinical and neuropsychological assessment batteries. J Gerontol B Psychol Sci Soc Sci 2002;57:P47-P53.

8. Rascovsky K, Hodges JR, Knopman D, Mendez MF, Kramer JH, Neuhaus J, et al. Sensitivity of revised diagnostic criteria for the behavioural variant of frontotemporal dementia. Brain 2011;134:2456-2477.
9. McKeith IG, Dickson DW, Lowe J, Emre M, O'Brien JT, Feldman H, et al. Diagnosis and management of dementia with Lewy bodies: third report of the DLB Consortium. Neurology 2005;65:1863-1872.

10. Román GC, Tatemichi TK, Erkinjuntti T, Cummings JL, Masdeu JC, Garcia JH, et al. Vascular dementia: diagnostic criteria for research studies. Report of the NINDS-AIREN International Workshop. Neurology 1993;43:250-260.

11. Winblad B, Palmer K, Kivipelto M, Jelic V, Fratiglioni L, Wahlund LO, et al. Mild cognitive impairment--beyond controversies, towards a consensus: report of the International Working Group on Mild Cognitive Impairment. J Intern Med 2004;256:240-246.

12. Emre M, Aarsland D, Brown R, Burn DJ, Duyckaerts C, Mizuno Y, et al. Clinical diagnostic criteria for dementia associated with Parkinson's disease. Mov Disord 2007;22:1689-1707.

13. American Psychiatric Association. Diagnostic and Statistical Manual of Mental Disorders (DSM- $5^{\circledR}$ ). Washington DC: American Psychiatric Pub; 2013.

14. McKhann GM, Knopman DS, Chertkow H, Hyman BT, Jack CR Jr, Kawas $\mathrm{CH}$, et al. The diagnosis of dementia due to Alzheimer's disease: recommendations from the National Institute on Aging-Alzheimer's Association workgroups on diagnostic guidelines for Alzheimer's disease. Alzheimers Dement 2011;7:263-269.

15. Sabri O, Seibyl J, Rowe C, Barthel H. Beta-amyloid imaging with florbetaben. Clin Transl Imaging 2015;3:13-26.

16. Vizamyl ${ }^{\mathrm{TM}}$ Flutemetamol F 18 Injection. Available at: https://www.accessdata.fda.gov/drugsatfda_docs/label/2017/203137s008lbl.pdf. Accessed September 22, 2020

17. Bahar-Fuchs A, Villemagne V, Ong K, Chetélat G, Lamb F, Reininger $\mathrm{CB}$, et al. Prediction of amyloid- $\beta$ pathology in amnestic mild cognitive impairment with neuropsychological tests. J Alzheimers Dis 2013; 33:451-462.

18. Burnham SC, Faux NG, Wilson W, Laws SM, Ames D, Bedo J, et al. A blood-based predictor for neocortical A $\beta$ burden in Alzheimer's disease: results from the AIBL study. Mol Psychiatry 2014;19:519-526.

19. Nakamura A, Kaneko N, Villemagne VL, Kato T, Doecke J, Doré V, et al. High performance plasma amyloid- $\beta$ biomarkers for Alzheimer's disease. Nature 2018;554:249-254.

20. Park JC, Han SH, Cho HJ, Byun MS, Yi D, Choe YM, et al. Chemically treated plasma $A \beta$ is a potential blood-based biomarker for screening cerebral amyloid deposition. Alzheimers Res Therapy 2017;9:20.

21. Ovod V, Ramsey KN, Mawuenyega KG, Bollinger JG, Hicks T, Schneider T, et al. Amyloid $\beta$ concentrations and stable isotope labeling kinetics of human plasma specific to central nervous system amyloidosis. Alzheimers Dement 2017;13:841-849.

22. Haghighi M, Smith A, Morgan D, Small B, Huang S. Identifying costeffective predictive rules of amyloid- $\beta$ level by integrating neuropsychological tests and plasma-based markers. J Alzheimers Dis 2015;43: 1261-1270.

23. Lee JH, Byun MS, Yi D, Sohn BK, Jeon SY, Lee Y, et al. Prediction of cerebral amyloid with common information obtained from memory clinic practice. Front Aging Neurosci 2018;10:309.

24. Wisniewski T, Drummond E. APOE-amyloid interaction: therapeutic targets. Neurobiol Dis 2020;138:104784.

25. Vemuri P, Knopman DS, Lesnick TG, Przybelski SA, Mielke MM, Graff-Radford J, et al. Evaluation of amyloid protective factors and alzheimer disease neurodegeneration protective factors in elderly individuals. JAMA Neurol 2017;74:718-726.

26. Power MC, Schneider AL, Wruck L, Griswold M, Coker LH, Alonso A, et al. Life-course blood pressure in relation to brain volumes. Alzheimers Dement 2016;12:890-899.

27. Glodzik L, Mosconi L, Tsui W, de Santi S, Zinkowski R, Pirraglia E, et al. Alzheimer's disease markers, hypertension, and gray matter damage in normal elderly. Neurobiol Aging 2012;33:1215-1227.

28. Wiseman RM, Saxby BK, Burton EJ, Barber R, Ford GA, O’Brien JT. Hippocampal atrophy, whole brain volume, and white matter lesions in older hypertensive subjects. Neurology 2004;63:1892-1897. 
Supplementary Table 1. Youden index, sensitivity, specificity, PPV, and NPV in all subjects

\begin{tabular}{cccccc}
\hline Cutoff & Youden index & Sensitivity & Specificity & PPV & NPV \\
\hline 0.1 & 1.005 & 1 & 0.005 & 0.543 & 1 \\
0.2 & 1.094 & 0.982 & 0.112 & 0.566 & 0.840 \\
0.3 & 1.273 & 0.932 & 0.340 & 0.625 & 0.810 \\
0.4 & 1.333 & 0.806 & 0.527 & 0.668 & 0.697 \\
0.5 & 1.385 & 0.694 & 0.691 & 0.726 & 0.657 \\
$0.556^{*}$ & 1.452 & 0.676 & 0.777 & 0.781 & 0.670 \\
0.6 & 1.423 & 0.631 & 0.793 & 0.782 & 0.645 \\
0.7 & 1.394 & 0.500 & 0.894 & 0.847 & 0.602 \\
0.8 & 1.267 & 0.320 & 0.947 & 0.877 & 0.541 \\
0.9 & 1.125 & 0.131 & 0.995 & 0.967 & 0.492 \\
\hline
\end{tabular}

The results of exploration on how the Youden index, sensitivity, specificity, PPV, and NPV change as the cutoff point of probability for a subject to be amyloid positive increases from 0.1 to 0.9 by 0.1 are presented as percentage values. *Best cut-off Prediction based on Youden Index. $\mathrm{PPV}$, positive predictive value; NPV, negative predictive value 
Supplementary Table 2. Youden index, sensitivity, specificity, PPV, and NPV in MCl subjects

\begin{tabular}{|c|c|c|c|c|c|}
\hline Cutoff & Youden index & Sensitivity & Specificity & PPV & NPV \\
\hline 0.1 & 1 & 1 & $\overline{0}$ & 0.541 & \\
\hline 0.2 & 1.006 & 0.996 & 0.011 & 0.543 & 0.667 \\
\hline 0.3 & 1.031 & 0.919 & 0.112 & 0.550 & 0.538 \\
\hline 0.4 & 1.227 & 0.833 & 0.394 & 0.619 & 0.667 \\
\hline 0.5 & 1.397 & 0.599 & 0.798 & 0.778 & 0.628 \\
\hline $0.504^{*}$ & 1.402 & 0.599 & 0.803 & 0.782 & 0.629 \\
\hline 0.6 & 1.366 & 0.536 & 0.830 & 0.788 & 0.602 \\
\hline 0.7 & 1.360 & 0.509 & 0.851 & 0.801 & 0.595 \\
\hline 0.8 & 1.240 & 0.293 & 0.947 & 0.867 & 0.531 \\
\hline
\end{tabular}

The results of exploration on how the Youden index, sensitivity, specificity, PPV, and NPV change as the cutoff point of probability for a subject to be amyloid positive increases from 0.1 to 0.9 by 0.1 are presented as percentage values. *Best cut-off Prediction based on Youden Index. PPV, positive predictive value; NPV, negative predictive value; $\mathrm{MCI}$, mild cognitive impairment 
Supplementary Table 3. Youden index, sensitivity, specificity, PPV, and NPV in AD subjects

\begin{tabular}{cccccl}
\hline Cut off & Youden index & Sensitivity & Specificity & PPV & NPV \\
\hline 0.1 & 1.036 & 1 & 0.036 & 0.759 & 1 \\
0.2 & 1.095 & 0.988 & 0.107 & 0.771 & 0.750 \\
0.3 & 1.274 & 0.988 & 0.286 & 0.808 & 0.889 \\
0.4 & 1.334 & 0.976 & 0.357 & 0.822 & 0.833 \\
0.5 & 1.405 & 0.941 & 0.464 & 0.842 & 0.722 \\
0.6 & 1.513 & 0.871 & 0.643 & 0.881 & 0.621 \\
$0.691^{*}$ & 1.574 & 0.788 & 0.786 & 0.918 & 0.550 \\
0.7 & 1.550 & 0.765 & 0.786 & 0.915 & 0.524 \\
0.8 & 1.456 & 0.671 & 0.786 & 0.905 & 0.440 \\
0.9 & 1.447 & 0.482 & 0.964 & 0.976 & 0.380 \\
\hline
\end{tabular}

The results of exploration on how the Youden index, sensitivity, specificity, PPV, and NPV change as the cutoff point of probability for a subject to be amyloid positive increases from 0.1 to 0.9 by 0.1 are presented as percentage values. *Best cut-off Prediction based on Youden Index. $\mathrm{PPV}$, positive predictive value; NPV, negative predictive value; $\mathrm{AD}$, Alzheimer's disease 